\title{
Subgrid and interelement interactions affect discretisations of stochastically forced diffusion
}

\author{
A. J. Roberts ${ }^{1}$
}

(Received 28 June 2006; revised 29 June 2007)

\begin{abstract}
Constructing discrete models of stochastic partial differential equations is very delicate. I apply dynamical systems theory to support spatial discretisations of the stochastically forced diffusion equation. To apply stochastic centre manifold theory, divide the physical domain into finite sized elements by introducing insulating internal boundaries which are later removed to fully couple the dynamical interactions between neighbouring elements. The approach automatically parametrises the stochastically forced microscale, subgrid structures within each element. The crucial aspect of this work is that we explore how a multitude of noise processes interact within and between neighbouring elements. Noise processes with coarse structure across a finite element are the most significant noises for the discrete model. Their influence also diffuses away to weakly correlate the noise in a spatial discretisation.
\end{abstract}

See http://anziamj.austms.org.au/ojs/index.php/ANZIAMJ/article/view/36 for this article, (c) Austral. Mathematical Soc. 2007. Published August 31, 2007. ISSN 1446-8735 


\section{Contents}

1 Introduction

C170

2 Stochastic centre manifold theory underpins modelling $\quad$ C173 2.1 A stochastic slow manifold exists . . . . . . . . . . . C174

2.2 The stochastic slow manifold captures the dynamics . . . . C176

3 Construct a memoryless discretisation of diffusion $\quad$ C177 3.1 Iteration converges to the asymptotic series . . . . . . . . C177

3.2 Corrections from a simple residual . . . . . . . . . . C C178

3.3 Some convolutions need to be separated . . . . . . . . C C180

3.4 Diffusion correlates noise across elements . . . . . . . . . C181

4 Conclusion

C185

References

C186

\section{Introduction}

We explore the nondimensional stochastically forced diffusion equation (SFDE)

$$
\frac{\partial u}{\partial t}=\frac{\partial^{2} u}{\partial x^{2}}+\sigma \phi(x, t),
$$

for a field $u(x, t)$ evolving in time $t$ in one spatial dimension $x$. The SFDE (1) has solutions forced by the stochastic noise $\phi(x, t)$ controlled by the strength parameter $\sigma$, and dissipated by diffusion, $u_{x x}$. Figure 1 shows the typical seething microscale structures in one simulation of the SFDE (1). We test a methodology for closure of macroscale spatial discretisations of the SFDE (1) through analysing and rationally modelling the microscale stochastic dynamics within and between spatial elements. 


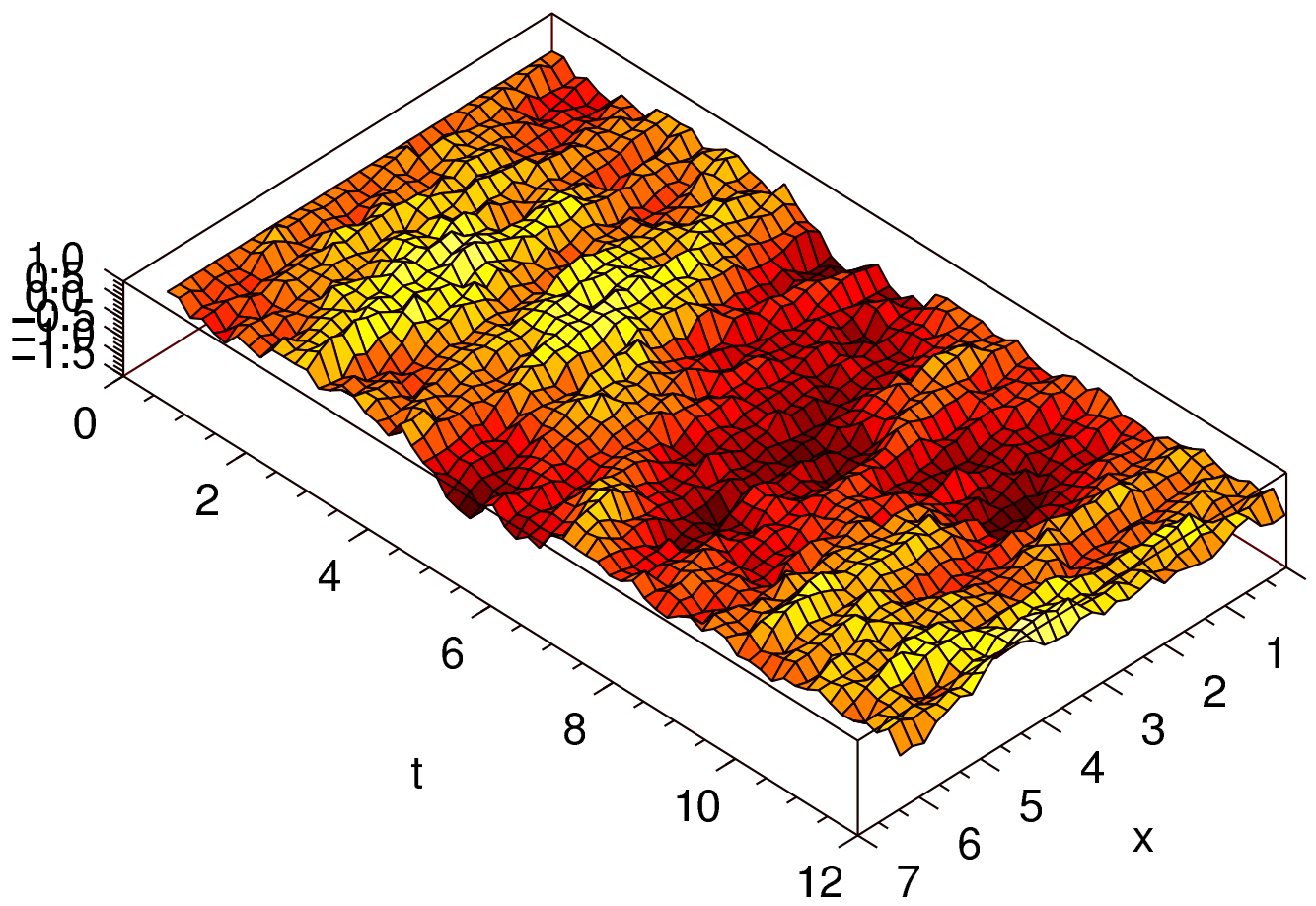

FiguRE 1: microscale simulation of one realisation of the stochastically forced diffusion $\operatorname{SFDE}(1)$, with noise intensity $\sigma=1$. This simulation uses the $\operatorname{SDE}(2)$ on a fine space-time mesh with $\delta x=\pi / 16$ and $\delta t=0.01$ but plotted every 19th time step. 
There are tricky subtleties. For example, the simplest finite difference approximation in space of the $\operatorname{SFDE}(1)$ on a regular grid in $\mathbf{x}$, say $X_{j}=j h$ for some constant grid spacing $h$, is

$$
\dot{\mathrm{u}}_{j}=\frac{\mathrm{u}_{j+1}-2 \mathrm{u}_{j}+\mathrm{U}_{j-1}}{\mathrm{~h}^{2}}+\sigma \phi\left(\mathrm{X}_{\mathrm{j}}, \mathrm{t}\right),
$$

where an over-dot denotes the time derivative $d / d t$, and $U_{j}(t)=u\left(X_{j}, t\right)$ is the value of the field $u(x, t)$ at the grid points $X_{j}$. Finite element approximations are similar. However, the analysis of Section 3 recommends we use instead

$$
\begin{aligned}
\dot{u}_{j} \approx & \frac{u_{j+1}-2 u_{j}+u_{j-1}}{h^{2}}+\sigma \psi_{j} \\
& -\frac{\sigma}{24}\left(\psi_{j+1}-2 \psi_{j}+\psi_{j-1}\right)+\frac{\sigma}{12 \sqrt{10}}\left(\hat{\psi}_{j+1}-2 \hat{\psi}_{j}+\hat{\psi}_{j-1}\right),
\end{aligned}
$$

see (21), for some independent noise processes $\psi_{j}$ and $\hat{\psi}_{j}$. The rationale is that the spatial diffusion on the subgrid scale between the grid points weakly correlates the noise that should be applied to each grid value. Thus the point sample $\phi_{j}$ of the noise $\phi$ in the simple model SDE (2) should be modified to spread a little across neighbouring elements; additionally, a small independent component $\hat{\psi}_{j}$ is also distributed over each triple of neighbouring grid values. Accounting explicitly for subgrid microscale physical processes resolves interaction between noise and diffusion to give the model SDE (3).

Stochastic centre manifold (SCM) theory supports the long time, macroscale modelling of detailed stochastic microscale dynamics [2, 6, e.g.]. Section 2 applies the SCM theory of Boxler [2] to support the discrete model SDE (3) of the SFDE (1). The methodology develops that used to rationally discretise deterministic PDEs $[9,10,11,7]$. The fiendish complication discussed in Section 3 is to account for noise and its dynamics which are distributed independently across space as well as time, both within an element and between neighbouring elements.

We discuss the forcing $\phi(x, t)$ as delta correlated in both space and time. However, most of the analysis also holds for deterministic forcing $\phi(x, t)$. 
Nonetheless, for definiteness, interpret all noise processes and all SDEs in the Stratonovich sense so that the rules of traditional calculus apply.

\section{Stochastic centre manifold theory underpins modelling}

I describe one way to place the spatial discretisation of SPDEs within the purview of stochastic centre manifold (SCM) theory. Then SCM theory assures us of the existence and relevance of the discrete model constructed Section 3. The SFDE (1) serves as a definite example of a broad class of dissipative SPDEs. The same methodology will be applied to nonlinear SPDEs.

Discretise space by the following artifice. For definite theoretical statements, suppose the field is L-periodic in the space coordinate $x$; that is, $\mathfrak{u}(x+L, t)=u(x, t)$. Divide one period of the domain into $m$ elements of equal and finite length $h=L / m$. Place equi-spaced grid points at $X_{j}$ at the centre of each element so that the $j$ th element is notionally $\left|x-X_{j}\right|<h / 2$. Mathematically we actually form the elements by introducing the artificial internal coupling conditions (ICCs)

$$
u_{j}\left(X_{j \pm 1}, t\right)-u_{j}\left(X_{j}, t\right)=\gamma\left[u_{j \pm 1}\left(X_{j \pm 1}, t\right)-u_{j}\left(X_{j}, t\right)\right],
$$

where $u_{j}(x, t)$ denotes the subgrid scale field of the $j$ th element - an element analytically extended to the neighbouring grid points $x=X_{j \pm 1}$. The coupling parameter $\gamma$ controls the flow of information between adjacent elements: when $\gamma=0$, adjacent elements are decoupled; when $\gamma=1$, the field in the jth element must extrapolate to the neighbouring elements' field at their grid point. These ICCs ensure, to high order in small element size $h$, that the discrete models are consistent with linear deterministic PDEs [10].

Base the discrete SCM modelling upon the dynamics when: firstly, the noise is absent, $\sigma=0$; and secondly, each element is decoupled from its 
neighbours, $\gamma=0$. When $\sigma=\gamma=0$ the dynamics of SFDE (1) with ICCs (4) reduce to that of deterministic linear diffusion within each element insulated from its neighbours:

$$
\frac{\partial u}{\partial t}=\frac{\partial^{2} u}{\partial x^{2}} \quad \text { such that } \pm\left. h \frac{\partial u_{j}}{\partial x}\right|_{x=x_{j} \pm h / 2}=0 .
$$

The discrete ICC (4) linearises to the above element boundary conditions (5) as the discrete ICC (4) is equivalent to [9]

$$
\pm\left.\mathrm{h} \frac{\partial u_{j}}{\partial x}\right|_{x=x_{j} \pm h / 2}=\gamma\left[\left.\mathcal{A} u_{j \pm 1}\right|_{x=x_{j \pm 1}}-\left.\mathcal{A} u_{j}\right|_{x=x_{j}}\right],
$$

where the near identity operator

$$
\mathcal{A}=\frac{ \pm h \partial_{x}}{\exp \left( \pm h \partial_{x}\right)-1}=1 \mp \frac{1}{2} \partial_{x}+\frac{1}{12} \partial_{x}^{2}-\frac{1}{720} \partial_{x}^{4}+\frac{1}{30240} \partial_{x}^{6}+\mathcal{O}\left(\partial_{x}^{8}\right) .
$$

All discussions of theoretical support for the discretisation use the element ICCs (6) instead of the computationally convenient ICCs (4). Although the analysis is based upon the near trivial parameter values $\sigma=\gamma=0$, stochastic centre manifold theory consequently supports the modelling of the dynamics at finite noise, $\sigma \neq 0$, and finite interelement coupling, $\gamma \neq 0$.

\subsection{A stochastic slow manifold exists}

The spectrum of the base diffusion problem (5) ensures a discretisation exists. In each element, (5) has solutions composed of a linear combination of modes $u \propto \operatorname{csn} k \theta \exp \left(-\beta_{k} t\right)$ where: firstly, the Fourier modes

$$
\operatorname{csn} k \theta= \begin{cases}\cos k \theta, & \text { for even } k, \\ \sin k \theta, & \text { for odd } k\end{cases}
$$

secondly, $\theta=\pi\left(x-X_{j}\right) / h$ measures subgrid position relative to the grid point within each element- the jth element is $|\theta| \leq \pi / 2$ although we do 
analytically extend the element to $|\theta| \leq \pi$; thirdly, the integer $k$ is the subgrid scale wavenumber; and lastly, the kth mode decays with rate

$$
\beta_{k}=\frac{\pi^{2} k^{2}}{h^{2}}, \quad k=0,1,2, \ldots
$$

The $k=0$ mode, that $u$ is constant in each element, is linearly neutral as its decay rate $\beta_{0}=0$. These neutral modes, one from each element, form the linear basis of the long term, discrete model.

Decompose the noise within each element as a linear combination of the fundamental Fourier modes (7):

$$
\phi(x, t)=\sum_{k=0}^{\infty} \phi_{j, k}(t) \operatorname{csn} k \theta, \quad \text { for }\left|x-X_{j}\right|<h / 2,
$$

where $\phi_{j, k}$ denotes the noise process of the kth wavenumber in the $j$ th element. Assume the set of processes $\left\{\phi_{j, k}\right\}$ are independent.

As the linear PDE (5) has $m$ eigenvalues of zero and all the other eigenvalues are negative, namely the stable eigenvalues $\leq-\beta_{1}=-\pi^{2} / h^{2}$, then, after adjoining the two trivial DEs $\mathrm{d} \sigma / \mathrm{dt}=\mathrm{d} \gamma / \mathrm{dt}=0$, SCM theory [2, Theorem 5.1 and 6.1] assures us that in some finite neighbourhood of $(u, \sigma, \gamma)=0$ there exists an $m+2$ dimensional stochastic slow manifold (SSM) where the field in the jth element is $u=u_{j}(\mathbf{u}(t), x, t, \sigma, \gamma)$, and where the jth component $\mathbf{U}_{j}$ of vector $\mathbf{U}$ measures the amplitude of the neutral mode in the jth element. ${ }^{1}$ For example, a low order approximation to the SSM is simply deterministic Lagrangian interpolation within each element:

$$
u_{j}=u_{j}+\gamma \frac{1}{2}(\theta / \pi)\left(u_{j+1}-u_{j-1}\right)+\gamma \frac{1}{2}(\theta / \pi)^{2}\left(u_{j+1}-2 u_{j}+u_{j-1}\right)+\cdots
$$

On the SSM the amplitudes $\mathbf{u}_{j}$ evolve according to $\dot{u}_{j}=g_{j}(\mathbf{u}, t, \sigma, \gamma)$ for some function $\mathrm{g}_{\boldsymbol{j}}$ such as the discrete $\operatorname{SDE}(3)$.

${ }^{1}$ The two extra dimensions of the SSM arise from the dependence upon the two parameters $\sigma$ and $\gamma$. 
Unfortunately, there is a caveat: Boxler's [2] theory is so far developed only for finite dimensional systems which satisfy a Lipschitz condition. Here, the SFDE (1) is infinite dimensional. There is some relevant but not yet directly applicable infinite dimensional theory [1, 5, e.g.] Future theoretical developments should rigorously support this approach.

\subsection{The stochastic slow manifold captures the dynamics}

The second key theorem of SCMs is that the evolution on the SSM, such as that described by the SDE (3), does capture the long term dynamics of the original SFDE (1). The Stochastic Relevance Theorem 7.1(i) [2] assures us that all nearby solutions of the SPDE (1) exponentially quickly in time approach the SSM $\mathfrak{u}=\mathfrak{u}_{\mathfrak{j}}(\mathbf{U}(\mathbf{t}), \boldsymbol{x}, \mathbf{t}, \sigma, \gamma)$, such as the low accuracy approximation (10). But crucially the theorem also assures us that the evolution of the trajectories approaching the SSM also approaches, on a cross-element diffusion time, the evolution of a trajectory on the SSM. That is, the evolution on the SSM, such as the $\operatorname{SDE}(3)$, faithfully describes the evolution of all solutions of SFDE (1) in some neighbourhood of the SSM. (In deterministic systems this property has been called "asymptotic completeness" [13].) Thus, in this context, the SSM model forms a discrete model that describes all the dynamics of SFDE (1) apart from exponentially decaying transients. This amazing theoretical support for the model holds at finite element size $h$.

However, there are two significant caveats. Firstly, although the asymptotic series are global in the grid value amplitudes $\mathcal{U}_{j}$, they are local in the parameters $\sigma$ and $\gamma$. Thus the rigorous theoretical support only applies in some finite neighbourhood of $\sigma=\gamma=0$. Secondly, the discretisations we develop and discuss have an error due to the finite truncation of the asymptotic series in the noise $\sigma$ and coupling $\gamma$. 


\section{Construct a memoryless discretisation of diffusion}

We explore how to construct a SSM, discrete model of the SFDE (1). The resulting models show how diffusion of noise processes, within and between adjacent elements, spatially correlate noise in the discretisation, see the SDE (3).

\subsection{Iteration converges to the asymptotic series}

The SCM approach establishes that the long term dynamics of the SFDE (1) may be parametrised by the grid value $\mathbf{U}_{j}$. However, most analyses of SDEs generate models with convolutions over fast time scales of the noise $[12, \S 2$, e.g.]. Here we greatly simplify the discretisation by removing such 'memory' convolutions [3, 4, 12, 14].

Consider the task of iteratively constructing a stochastic model for the SPDE (1). We seek solutions of the SPDE such that within the jth element the field is described by some asymptotic expression, $\mathfrak{u}=\mathfrak{u}_{\mathfrak{j}}(\mathbf{U}, x, t, \sigma, \gamma)=$ $\mathbf{U}_{j}+\cdots$, such that the vector of amplitudes $\mathbf{U}$ evolve according to some prescription that we also only know asymptotically, $\dot{U}_{j}=g_{j}(\mathbf{U}, t, \sigma, \gamma)$, such as the SDE (3). The steps in the construction of the asymptotic approximations proceed iteratively. Suppose that at some stage we have some asymptotic approximation to the model, then the next iteration is to seek small corrections, denoted $u_{j}^{\prime}$ and $g_{j}^{\prime}$. Determine the small corrections from a linear equation (building upon the well established detailed derivation for deterministic systems [8, e.g.], the only substantive change is the recognition of direct time dependence in the small correction $\partial u_{j}^{\prime} / \partial t$ ): first, substitute $u=u_{j}+u_{j}^{\prime}$ and $\dot{\mathrm{u}}_{j}=\mathrm{g}_{j}+\mathrm{g}_{j}^{\prime}$ into the SFDE (1); second, linearise the problem for $\mathfrak{u}_{j}^{\prime}$ and $\mathrm{g}_{j}^{\prime}$ by dropping products of small corrections; third, simplify by replacing coefficients of corrections by their leading order approximation; and thus lastly, 
obtain that the corrections should satisfy

$$
\frac{\partial u_{j}^{\prime}}{\partial t}-\frac{\partial^{2} u_{j}^{\prime}}{\partial x^{2}}+g_{j}^{\prime}=\operatorname{residual}_{(1)} \text {. }
$$

Here the "residual" is the residual of the SFDE (1) evaluated for the currently known asymptotic approximation. In addition, the inter-element ICCs (4) provide boundary conditions for $\mathfrak{u}_{\mathfrak{j}}^{\prime}$, namely

$$
u_{j}^{\prime}\left(X_{j \pm 1}, t\right)-u_{j}^{\prime}\left(X_{j}, t\right)+\operatorname{residual}_{(4)}=0 .
$$

For example, suppose at some stage we had found the deterministic part of the model in the $j$ th element was that of classic Lagrangian interpolation

$$
\begin{array}{ll} 
& u_{j}(x, t)=u_{j}+\gamma\left[\frac{1}{2}(\theta / \pi)^{2} \delta^{2}+(\theta / \pi) \mu \delta\right] u_{j}+\mathcal{O}\left(\sigma+\gamma^{2}\right) \\
\text { such that } & \dot{u}_{j}=\frac{\gamma}{h^{2}} \delta^{2} u_{j}+\mathcal{O}\left(\sigma+\gamma^{2}\right)
\end{array}
$$

where hereafter the discrete difference and mean operators reduce the algebraic length of expressions, respectively $\delta \mathrm{U}_{j}=\mathrm{U}_{j+1 / 2}-\mathrm{U}_{j-1 / 2}$ and $\mu \mathrm{U}_{j}=$ $\frac{1}{2}\left(u_{j+1 / 2}+u_{j-1 / 2}\right)$. Then in the next iteration, using (13), the residual of the SFDE (1) is

$$
\operatorname{residual}_{(1)}=-\frac{\gamma^{2}}{h^{2}}\left[(\theta / \pi) \mu \delta^{3}+\frac{1}{2}(\theta / \pi)^{2} \delta^{4}\right] u_{j}+\sigma \sum_{k=0}^{\infty} \phi_{j, k}(t) \operatorname{csn} k \theta
$$

whereas the ICCs have residual $_{(4)}=0$.

\subsection{Corrections from a simple residual}

Now explore how to solve (11-12) for corrections given some residual such as (14). The terms in the residual split into two categories: 
- For each component of the form $\psi(t) \operatorname{csn} k \theta$, for wavenumber $k \geq 1$, include a corresponding component in the correction of $u_{j}^{\prime}=\mathcal{Z}_{k} \psi(t) \operatorname{csn} k \theta$ in which the operator $\mathcal{Z}_{\mathrm{k}}$ denotes convolution over the history:

$$
\mathcal{Z}_{k} \phi=\exp \left[-\beta_{k} t\right] \star \psi(t)=\int_{-\infty}^{t} \exp \left[-\beta_{k}(t-\tau)\right] \psi(\tau) d \tau .
$$

- But any component constant across the element, such as $\sigma \phi_{j, 0}(t)$ in residual (14), must cause a contribution to the evolution correction $\mathbf{g}_{\mathbf{j}}^{\prime}$, here simply $g_{j}^{\prime}=\sigma \phi_{j, 0}$, as no uniformly bounded component in $u_{j}^{\prime}$ can match a constant component in the residual - this is the standard solvability condition for singular perturbations.

For example, with the residual (14) the corresponding corrections $\mathbf{g}_{j}^{\prime}$ and $\mathfrak{u}_{j}^{\prime}$ improve the SSM model (13) to

$$
\begin{aligned}
\mathrm{u}_{\mathrm{j}}(\mathrm{x}, \mathrm{t})= & \mathrm{u}_{\mathrm{j}}+\gamma\left[\frac{1}{2}(\theta / \pi)^{2} \delta^{2}+(\theta / \pi) \mu \delta\right] \mathrm{u}_{j} \\
& +\gamma^{2}\left[\frac{1}{6}\left((\theta / \pi)^{3}-(\theta / \pi)\right) \mu \delta^{3}+\frac{1}{24}\left((\theta / \pi)^{4}-(\theta / \pi)^{2}\right) \delta^{4}\right] \mathrm{u}_{j} \\
& +\sigma \sum_{\mathrm{k}=1}^{\infty} \mathcal{Z}_{\mathrm{k}} \phi_{j, \mathrm{k}} \operatorname{csn} \mathrm{k} \theta+\mathcal{O}\left(\sigma^{3 / 2}+\gamma^{3}\right) \\
\dot{\mathrm{u}}_{j}= & \frac{\gamma}{\mathrm{h}^{2}} \delta^{2} \mathrm{u}_{j}-\frac{\gamma^{2}}{12 \mathrm{~h}^{2}} \delta^{4} \mathrm{u}_{\mathrm{j}}+\sigma \phi_{j, 0}+\mathcal{O}\left(\sigma^{3 / 2}+\gamma^{3}\right)
\end{aligned}
$$

The $\gamma^{2}$ correction modifies the deterministic terms of the model (13) to (when fully coupled, $\gamma=1$ ) classic finite difference expressions of fourth order consistency as element size $h \rightarrow 0$. The noise induced $\sigma \phi_{j, 0}$ term straightforwardly represent the mean forcing in the jth element. It is the next iteration that begins to account for interesting nontrivial subgrid scale stochastic processes within the finite sized elements. 


\subsection{Some convolutions need to be separated}

A more delicate issue arises in subsequent corrections. The next iteration uses (16), whence the ICCS residual $(4)=\gamma \sigma \sum_{k=2, \text { even }}^{\infty}\left[\mathcal{Z}_{k} \phi_{j, k}-\mathcal{Z}_{k} \phi_{j \pm 1, k}\right]+$ $\mathcal{O}\left(\sigma^{3}+\gamma^{3}\right)$. Satisfy the ICCs (12) with this residual $(4)$ by incorporating into the approximate SSM field (16) the following subgrid field correction $u_{j}^{\prime}=+\gamma \sigma\left[(\theta / \pi) \mu \delta+\frac{1}{2}(\theta / \pi)^{2} \delta^{2}\right] \sum_{k=2, \text { even }}^{\infty} \mathcal{Z}_{k} \phi_{j, k}$. Then using (17) and the Fourier expansion of this correction added to the right-hand side of (16), the SFDE (1) has

$$
\begin{aligned}
& \text { residual }_{(1)}=\gamma \sigma \delta^{2} \sum_{k=2, \text { even }}^{\infty}\left(\frac{1}{h^{2}}+\frac{\beta_{k}}{24}\right) \mathcal{Z}_{k} \phi_{j, k}-\gamma \sigma \frac{1}{24} \delta^{2} \sum_{k=0, \text { even }}^{\infty} \phi_{j, k} \\
& -\gamma \sigma \frac{4}{\pi^{2}} \sum_{k=1, \text { odd }}^{\infty} \frac{(-1)^{\frac{k-1}{2}}}{k^{2}} \sin k \theta \mu \delta\left[\sum_{\ell=0, \text { even }}^{\infty} \phi_{j, \ell}-\sum_{\ell=2, \text { even }}^{\infty} \beta_{\ell} \mathcal{Z}_{\ell} \phi_{j, \ell}\right] \\
& -\gamma \sigma \frac{2}{\pi^{2}} \sum_{k=2, \text { even }}^{\infty} \frac{(-1)^{k / 2}}{k^{2}} \cos k \theta \delta^{2}\left[\sum_{\ell=0, \text { even }}^{\infty} \phi_{j, \ell}-\sum_{\ell=2, \text { even }}^{\infty} \beta_{\ell} \mathcal{Z}_{\ell} \phi_{j, \ell}\right] \\
& +\mathcal{O}\left(\sigma^{3}+\gamma^{3}\right) .
\end{aligned}
$$

The components of $\operatorname{csn} k \theta$ in the second and third line above just induce a corresponding component in the correction $\mathfrak{u}_{j}^{\prime}$ via a further convolution $\mathcal{Z}_{k}$. The components constant across the element, in the first line above, are the delicate issue. Part of these components can be integrated in time [4, 14, 3, 12]: since for any $\phi(t), \frac{\mathrm{d}}{\mathrm{dt}} \mathcal{Z}_{\mathrm{k}} \phi=-\beta_{\mathrm{k}} \mathcal{Z}_{\mathrm{k}} \phi+\phi$, from the stochastic convolution definition (15), thus $\mathcal{Z}_{\mathrm{k}} \phi=\frac{1}{\beta_{\mathrm{k}}}\left[-\frac{\mathrm{d}}{\mathrm{dt}} \mathcal{Z}_{\mathrm{k}} \phi+\phi\right]$, and so we separate such a convolution in the residual, when multiplied by the neutral mode of a constant across the element, into:

- the first part, $-\frac{\mathrm{d}}{\mathrm{dt}} \mathcal{Z}_{\mathrm{k}} \phi / \beta_{\mathrm{k}}$, which is integrated into the next update $\mathrm{u}_{\mathrm{j}}^{\prime}$ for the subgrid field;

- and the second part, $\phi / \beta_{k}$, which updates $g_{j}^{\prime}$ without introducing a fast-time memory convolution into the evolution. 
For the example residual (18) the terms in the first line thus force terms $-\gamma \sigma \delta^{2} \sum_{k=2 \text {,even }}^{\infty}\left(\frac{1}{\pi^{2} k^{2}}+\frac{1}{24}\right) \mathcal{Z}_{k} \phi_{j, k}$ into the subgrid field making the SSM

$$
\begin{aligned}
& u_{j}(x, t)=u_{j}+\gamma\left[\frac{1}{2}(\theta / \pi)^{2} \delta^{2}+(\theta / \pi) \mu \delta\right] u_{j} \\
& +\gamma^{2}\left[\frac{1}{6}\left((\theta / \pi)^{3}-(\theta / \pi)\right) \mu \delta^{3}+\frac{1}{24}\left((\theta / \pi)^{4}-(\theta / \pi)^{2}\right) \delta^{4}\right] \\
& +\sigma \sum_{k=1}^{\infty} \mathcal{Z}_{k} \phi_{j, k} \operatorname{csn} k \theta \\
& -\gamma \sigma \frac{4}{\pi^{2}} \sum_{k=1, \text { odd }}^{\infty} \frac{(-1)^{\frac{k-1}{2}}}{k^{2}} \sin k \theta \mu \delta \mathcal{Z}_{k}\left[\sum_{\ell=0, \text { even }}^{\infty} \phi_{j, \ell}-\sum_{\ell=2, \text { even }}^{\infty} \beta_{\ell} \mathcal{Z}_{\ell} \phi_{j, \ell}\right] \\
& -\gamma \sigma \frac{2}{\pi^{2}} \sum_{k=2, \text { even }}^{\infty} \frac{(-1)^{k / 2}}{k^{2}} \cos k \theta \delta^{2} \mathcal{Z}_{k}\left[\sum_{\ell=0, \text { even }}^{\infty} \phi_{j, \ell}-\sum_{\ell=2, \text { even }}^{\infty} \beta_{\ell} \mathcal{Z}_{\ell} \phi_{j, \ell}\right] \\
& -\gamma \sigma \delta^{2} \sum_{k=2 \text {,even }}^{\infty}\left(\frac{1}{\pi^{2} k^{2}}+\frac{1}{24}\right) \mathcal{Z}_{k} \phi_{j, k}+\mathcal{O}\left(\sigma^{3}+\gamma^{3}\right) .
\end{aligned}
$$

More interestingly, the terms in the first line of the example residual (18) also force a correction to the evolution to the model SDE

$$
\begin{aligned}
\dot{\mathrm{u}}_{j}= & \frac{\gamma}{\mathrm{h}^{2}} \delta^{2} \mathrm{u}_{j}-\frac{\gamma^{2}}{12 \mathrm{~h}^{2}} \delta^{4} \mathrm{u}_{j}+\sigma \phi_{j, 0} \\
& +\gamma \sigma \delta^{2}\left[-\frac{1}{24} \phi_{j, 0}+\frac{1}{\pi^{2}} \sum_{\mathrm{k}=2, \mathrm{even}}^{\infty} \frac{1}{\mathrm{k}^{2}} \phi_{j, k}\right]+\mathcal{O}\left(\sigma^{3}+\gamma^{3}\right) .
\end{aligned}
$$

The order of error in these approximations comes from the terms present in the residuals but so far ignored when determining corrections.

\subsection{Diffusion correlates noise across elements}

The SDE (20) shows, through the second difference terms $\delta^{2} \phi_{j, k}$, noise sources affect neighbouring elements. This effect arises because the noise in one 
element creates spatial structures that diffuse out into neighbouring elements and affect the evolution.

The asymptotic approximate SDE (20) models the forced diffusion dynamics of the SFDE (1) when we set the coupling parameter $\gamma=1$. Undesirably, the resultant model SDE has an infinite sum of noise components. Thus we combine the infinite sum of noise terms into just one unknown noise with the same statistics:

$$
\frac{1}{\pi^{2}} \sum_{k=2, \text { even }}^{\infty} \frac{1}{k^{2}} \phi_{j, k} \equiv \frac{1}{4 \pi^{2}} \sqrt{\sum_{n=1}^{\infty} \frac{1}{n^{4}}} \hat{\phi}_{j}(t)=\frac{1}{12 \sqrt{10}} \hat{\phi}_{j}(t)
$$

where the effectively new stochastic noise $\hat{\phi}_{j}(t)$ represents the cumulative effect of the infinite sum of the stochastic components $\phi_{j, k}$ for $k$ even. Thus the model (20) becomes, in the fully coupled case of $\gamma=1$,

$$
\dot{\mathrm{u}}_{j}=\frac{1}{\mathrm{~h}^{2}} \delta^{2} \mathrm{u}_{j}-\frac{1}{12 \mathrm{~h}^{2}} \delta^{4} \mathrm{u}_{j}+\sigma\left[\phi_{j, 0}-\frac{1}{24} \delta^{2} \phi_{j, 0}+\frac{1}{12 \sqrt{10}} \delta^{2} \hat{\phi}_{j}\right] .
$$

Instead of the infinite number of noise processes in (20), this model has only $2 \mathrm{~m}$ noise modes for a spatial domain with $\mathrm{m}$ elements.

The interesting and subtle component of the model (21) is $\frac{1}{12 \sqrt{10}} \delta^{2} \hat{\phi}_{j}$. Numerical simulations confirm this component exists on the macroscale. To see the effect most clearly the numerical simulation plotted in Figure 2 has noise components $\phi_{j, k}=0$ for $k=0$ and for odd $k$. Also, Figure 2 applies the noise $\phi=\sum_{2 \text {,even }}^{\infty} \phi_{1, k}(\mathrm{t}) \cos \mathrm{k} \theta$ to just one macroscale element, namely $0<x<\pi / 2$, to see how its influence spreads in space. See that although the noise induced fluctuations dominantly occur in the forced element, the noise has a weak effect outside the element. The component $\delta^{2} \hat{\phi}_{j}$ in (21) models this weak effect.

Simulations also confirm the amplitudes of the component $\frac{1}{12 \sqrt{10}} \delta^{2} \hat{\phi}_{j}$. Figure 3 plots two grid values of the field $u(x, t)$ as a function of time $t$ : one 


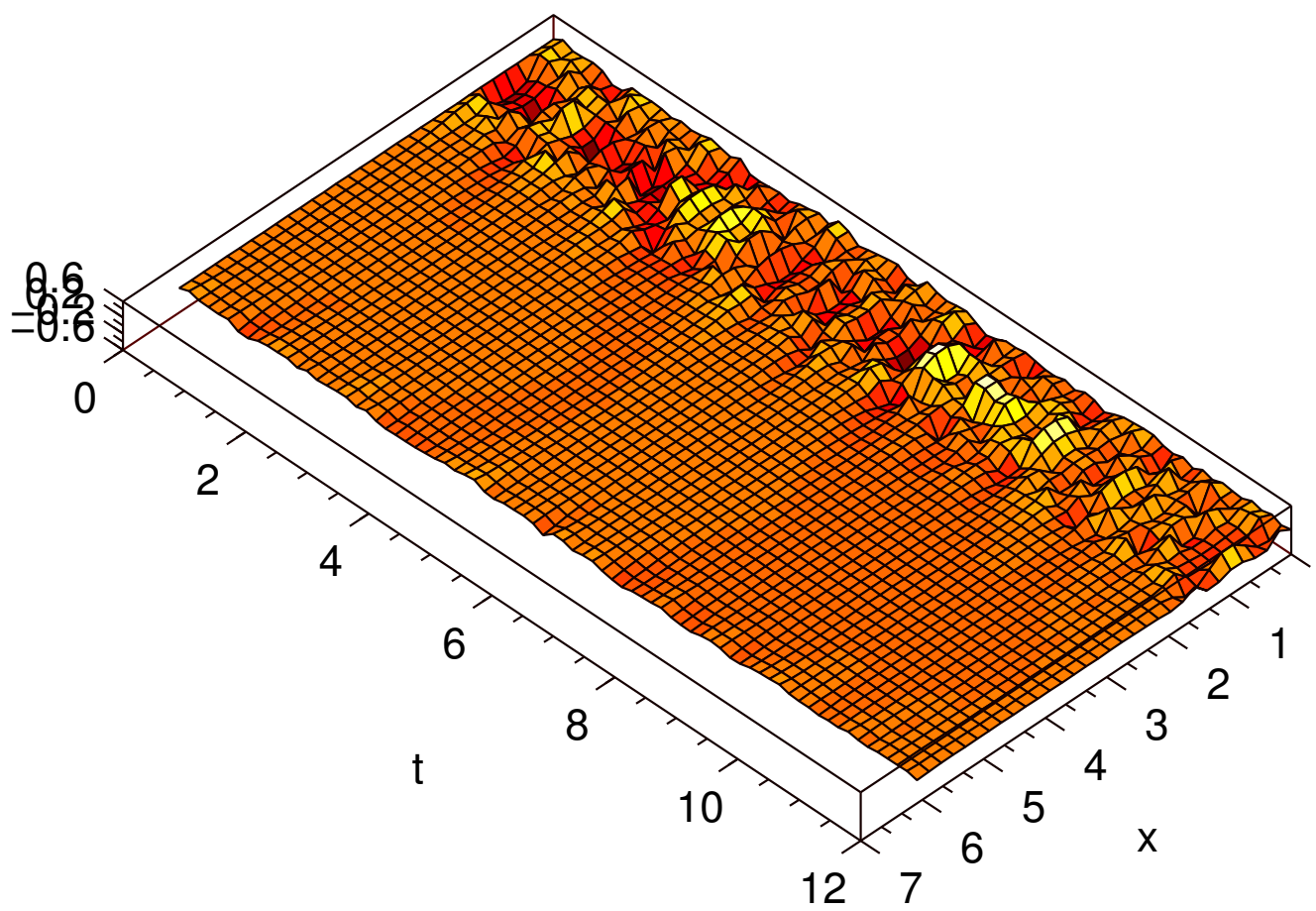

FiguRe 2: microscale simulation of the stochastically forced diffusion $\operatorname{SPDE}(1)(\delta x=\pi / 16$ and $\delta t=0.01$ on a $2 \pi$-periodic domain). Here restrict the noise $\phi(x, t)$ to one quarter of the domain, representing one macroscale element, and to the even modes, $\phi=\sum_{2, \text { even }}^{\infty} \phi_{1, k}(t) \cos k \theta$. A weak field diffuses out into the surrounding domain correlating the effective macroscale noise as in (21). 


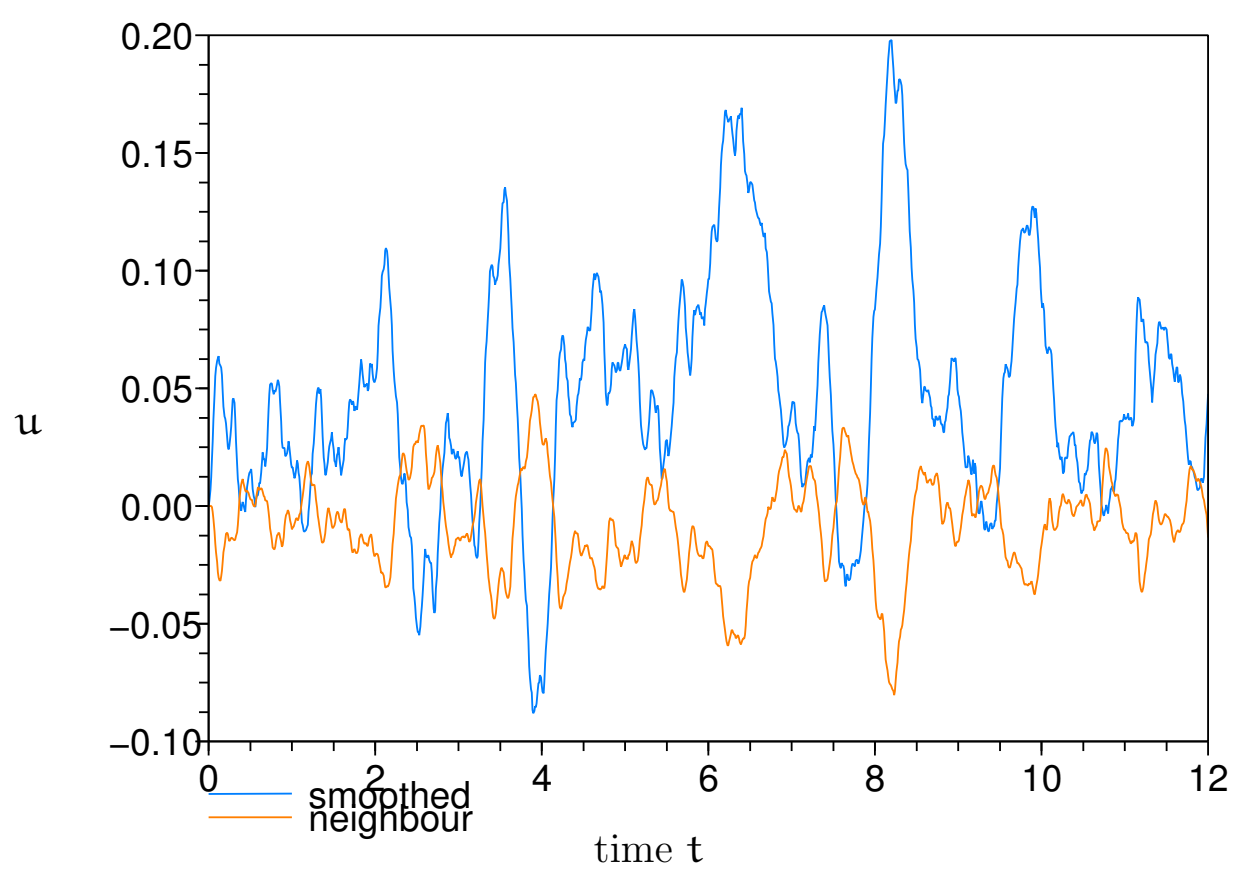

Figure 3: for the simulation shown in Figure 3, plot the smoothed "macroscale grid value" $\mathfrak{u}(\pi / 4, t)$ and the neighbouring "macroscale grid value" $u(3 \pi / 4, t)$ as a function of time. The microscale stochastic forcing in one element generates the effectively opposite forcing in the neighbouring elements as predicted by (21). 
at the centre $x=\frac{1}{4} \pi$ of the forced element, $0<x<\frac{1}{2} \pi$; and the other at the centre $x=\frac{3}{4} \pi$ of the adjacent element, $\frac{1}{2} \pi<x<\pi$. But recall that (21) models macroscale space-time dynamics. Thus I smooth the microscale time fluctuations of the grid value $u\left(\frac{1}{4} \pi, t\right)$ with the normalised operator $\hat{\mathcal{Z}}_{1}=\left[\mathcal{Z}_{1} 1\right]^{-1} \mathcal{Z}_{1}$ that smooths over an inter-element diffusion time. See in Figure 3 that the smoothed grid value of the forced element has very much the same shaped fluctuations, but opposite and roughly twice the size of the fluctuations in the neighbouring grid value. This is as predicted by the model (21). Furthermore, the model predicts the standard deviation of the the two grid values should be $1 / 6 \sqrt{10}=.0527$ and $1 / 12 \sqrt{10}=.0264$. The simulation shown in Figure 3 has standard deviations .050 and .021 , respectively, which are close enough considering the relatively few inter-element diffusion times simulated in the figure. Such quantitative agreement between the subtleties of the SSM modelling and the numerical simulations supports this approach to modelling SPDEs.

\section{Conclusion}

The particular example SFDE (1) demonstrates how to analyse the net effect of many independent subgrid stochastic effects both within an element and between neighbouring elements. SCM theory [2] supports the existence, relevance and order of accuracy of the discrete models.

Further research will explore the modelling of SPDEs: on domains with physical boundary conditions at their extremes [11, for PDEs]; in higher spatial dimensions [7, for PDEs]; to models of higher order in the coupling between elements; with the aid of computer algebra to handle the enormous details of the nonlinear subgrid dynamics and the inter-element interactions in nonlinear SPDEs; and the appearance in nonlinear SPDEs of effective mean drift and volatility effects of components quadratic in the noise [3, 12]. 


\section{References}

[1] D. Blomker, M. Hairer, and G. A. Pavliotis. Modulation equations: stochastic bifurcation in large domains. Communications in Mathematical Physics, 258:479-512, 2005. doi:10.1007/s00220-005-1368-8. C176

[2] P. Boxler. A stochastic version of the centre manifold theorem. Probab. Th. Rel. Fields, 83:509-545, 1989. C172, C175, C176, C185

[3] Xu Chao and A. J. Roberts. On the low-dimensional modelling of Stratonovich stochastic differential equations. Physica A, 225:62-80, 1996. doi:10.1016/0378-4371(95)00387-8. C177, C180, C185

[4] P. H. Coullet, C. Elphick, and E. Tirapegui. Normal form of a Hopf bifurcation with noise. Physics Letts, 111A(6):277-282, 1985. C177, C180

[5] Jinqiao Duan, Kening Lu, and Bjorn Schmalfuss. Invariant manifolds for stochastic partial differential equations. The Annals of Probability, 31:2109-2135, 2003. doi:10.1214/aop/1068646380. C176

[6] E. Knobloch and K. A. Wiesenfeld. Bifurcations in fluctuating systems: The centre manifold approach. J. Stat Phys, 33:611-637, 1983. C172

[7] T. MacKenzie and A. J. Roberts. Holistic discretisation of shear dispersion in a two-dimensional channel. In K. Burrage and Roger B. Sidje, editors, Proc. of 10th Computational Techniques and Applications Conference CTAC-2001, volume 44, pages C512-C530, March 2003. http://anziamj . austms .org. au/V44/CTAC2001/Mack. C172, C185

[8] A. J. Roberts. Low-dimensional modelling of dynamics via computer algebra. Computer Phys. Comm., 100:215-230, 1997. C177 
[9] A. J. Roberts. Holistic discretisation ensures fidelity to Burgers' equation. Applied Numerical Modelling, 37:371-396, 2001. C172, C174

[10] A. J. Roberts. A holistic finite difference approach models linear dynamics consistently. Mathematics of Computation, 72:247-262, 2002. http://www . ams .org/mcom/2003-72-241/S0025-5718-02-01448-5. C172, C173

[11] A. J. Roberts. Derive boundary conditions for holistic discretisations of Burgers' equation. In K. Burrage and Roger B. Sidje, editors, Proc. of 10th Computational Techniques and Applications Conference CTAC-2001, volume 44, pages C664-C686, March 2003. http://anziamj.austms.org.au/V44/CTAC2001/Robe. C172, C185

[12] A. J. Roberts. A step towards holistic discretisation of stochastic partial differential equations. In Jagoda Crawford and A. J. Roberts, editors, Proc. of 11th Computational Techniques and Applications Conference CTAC-2003, volume 45, pages C1-C15, December 2003. [Online] http://anziamj . austms.org.au/V45/CTAC2003/Robe [December 14, 2003]. C177, C180, C185

[13] J. C. Robinson. The asymptotic completeness of inertial manifolds. Nonlinearity, 9:1325-1340, 1996. http://www.iop.org/EJ/abstract/0951-7715/9/5/013. C176

[14] N. Sri Namachchivaya and Y. K. Lin. Method of stochastic normal forms. Int. J. Nonlinear Mechanics, 26:931-943, 1991. C177, C180 


\section{Author address}

1. A. J. Roberts, Computational Engineering and Science Research Centre, Department of Mathematics \& Computing, University of Southern Queensland, Toowoomba, Queensland 4352, Australia. mailto:aroberts@usq.edu.au 